\title{
The Strict Locality of Phonological Processes
}

\author{
Jane Chandlee*
}

LSA Annual Meeting, Minneapolis, January 2-5, 2014

1. Introduction This paper addresses the question of "what is a possible phonological process' from a computational perspective. Many previous studies have offered explanations for why certain processes are attested and/or common while others are unattested or rare (see Hume \& Johnson 2001, Hayes et al. 2004, Blevins 2004, among others). Following work on phonotactics by Heinz $(2007,2009,2010)$, the goal of the present study is to demonstrate the extent to which computational properties can distinguish the subset of what is phonologically possible from the larger set of logically possible processes.

Specifically, I identify a strong computational property of the mapping from underlying representation (UR) to surface representation (SR) in local phonological processes. This property is called Input Strict Locality (ISL) after the well-studied Strictly Local formal languages (McNaughton \& Papert 1971, Rogers \& Pullum 2011, Rogers et al. 2013). I demonstrate, based on a review of the P-Base database of phonological patterns (v1.95, Mielke 2008) that this property has broad empirical coverage and describe how, as a defining property of these mappings, Input Strict Locality has utility in phonological learning.

2. Phonological mappings The computational perspective adopted in this paper stems from the observation that both rule- and constraint-based theories of generative phonological grammars describe the same $\mathrm{UR} \mapsto \mathrm{SR}$ mappings. Consider an SPE-style (Chomsky \& Halle 1968) rewrite rule of the form in (1). This rule will map an underlying CAD sequence to CBD.

$$
\mathrm{A} \rightarrow \mathrm{B} / \mathrm{C} \_\mathrm{D}
$$

As discussed by Baković (2013), in a constraint-based grammatical framework like OT (Prince \& Smolensky 1993) or HG (Legendre et al. 1990) the same mapping from CAD to $\mathrm{CBD}$ is achieved by the ranking in (2), where ${ }^{*} \mathrm{CAD}$ is a markedness constraint and $\operatorname{FAITH}(\mathrm{A} \mapsto \mathrm{B})$ abbreviates the faithfulness constraint(s) violated when A surfaces as B.

$$
{ }^{*} \mathrm{CAD} \gg \mathrm{FAITH}(\mathrm{A} \mapsto \mathrm{B})
$$

The approach taken in this paper is to model this $\mathrm{UR} \mapsto \mathrm{SR}$ mapping as a string-to-string function $f$, with the goal of revealing properties of that mapping that are therefore independent of the grammatical formalism used to describe it. In particular, it is argued that Input Strict Locality is a defining property of the $f$ that models many phonological processes. In other words, these processes can be modeled with Input Strictly Local functions.

*Thanks to Jeffrey Heinz, James Rogers, Rémi Eyraud, Adam Jardine, and members of the UD Computational Linguistics Group for valuable questions and feedback. This work was supported by NSF grant CPS\#1035577. Author: Jane Chandlee, Center for Pediatric Auditory and Speech Sciences, Nemours Biomedical Research, Wilmington, DE (chandlee@asel.udel.edu). 
It is well-known that phonological rewrite rules of the form in (1) describe regular relations when A, B, C, and D are regular languages (Johnson 1984, Koskenniemi 1983, Kaplan \& Kay 1994). The ISL functions are a proper subset of the regular relations, and therefore the analysis presented here provides the strongest computational characterization of these processes to date.

3. Strict Locality The computational notion of Strict Locality comes from the class of formal languages known as Strictly Local languages (McNaughton \& Papert 1971, Rogers \& Pullum 2011, Rogers et al. 2013). What distinguishes these languages is that they can be defined with a grammar of contiguous substrings whose length is bounded by some integer $k$. A given string is in the language if and only if its own $k$-length substrings are a subset of the grammar. These languages have been shown to model natural language phonotactics provided that the markedness constraint can be represented by a contiguous substring of bounded length (Heinz 2010). For example, in a language with a constraint against D\# sequences (where D is a voiced obstruent), the set of well-formed surface strings can be modeled with a SL-2 grammar that allows all substrings of length 2 except for D\#.

This notion of locality is here applied to mappings by defining a particular type of finite state transducer (FST) that enforces a Strict Locality requirement on the kinds of mappings it can represent. More specifically, the Strict Locality requirement is applied to the input side of the mapping; hence the name Input Strictly Local. As an example, the ISL FST in Figure 1 describes the mapping of final devoicing (see Chandlee (2014) for a detailed presentation of these automata representations of processes). Briefly, starting in the $\lambda$-state (marked with a hexagon) the FST follows the transitions by reading an input string one segment at a time and produces output as indicated with the segment(s) after the colon on the transition labels (note $\lambda$ is the 'empty' string with no symbols). Any string ending in D will reach state $\mathrm{D}$, at which point that final $\mathrm{D}$ will be replaced by voiceless $\mathrm{T}$ (as dictated by the final transition on \#).

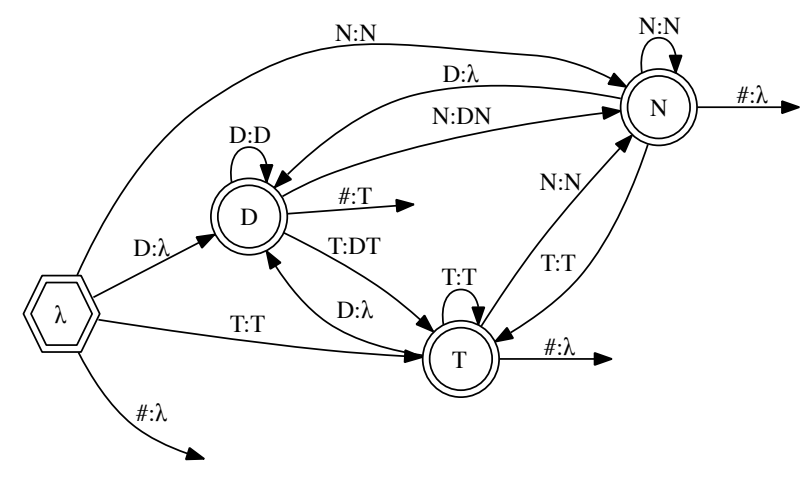

Figure 1: ISL FST for final devoicing

The properties that make Figure 1 an ISL FST are 1) the states correspond to all possible substrings up to length $k-1$ (in this example $k=2$ because the illegal sequence the process targets, D\#, is 2 segments long) and 2) the transitions are defined such that being in 
a state is only possible (and is required) if that state's label matches the most recent $k-1$ symbols of the input string.

A version of final devoicing that could not be described with such an FST is one in which the obstruent only devoices if the word contains an even number of segments. This logically possible but unattested (and odd) process could, however, be described with a non-ISL FST (i.e., it is still a regular relation). In this way, the Input Strict Locality requirement rules out non-phonological mappings.

4. Empirical coverage The central claim of this paper is that Input Strict Locality is a defining property of local phonological mappings. To support this claim empirically, a review was conducted of the approximately 5500 processes in the P-Base (v1.95, Mielke 2008) database of phonological patterns. A process was considered ISL if an ISL FST could be constructed to model its mapping. Based on this criterion, at least $96 \%$ of the patterns in the database are ISL. These processes include local substitution, deletion, and epenthesis, as well as synchronic metathesis. Beyond phonology proper, local partial reduplication and general affixation can also be shown to be ISL.

Of course this list of ISL processes has several notable exceptions, including long-distance processes like vowel harmony (with transparent vowels), long-distance assimilation and dissimilation, spreading processes, and certain tonal processes. Current and future work aims to show that these processes are also computationally restricted in desirable ways given a parallel notion of Output Strict Locality (see Chandlee (2014) for details).

5. Utility in learning In addition to its typological predictions, as a defining property of phonological mappings Input Strict Locality can be used as a bias or inductive principle of the phonological learner. One such learning algorithm has been implemented and proven to learn the class of ISL functions from positive data. Only a sketch of this algorithm is presented here; see Chandlee (2014) and Chandlee et al. (2014) for details and proofs.

The learner is given a set of input-output string pairs for the target mapping; continuing with the final devoicing example this set would include pairs like (VD,VT), (ND, $\mathrm{NT}),(\mathrm{TND}, \mathrm{TNT}),(\mathrm{NVN}, \mathrm{NVN})$, where again $\mathrm{D}$ is a voiced obstruent and $\mathrm{T}$ is a voiceless obstruent, $\mathrm{V}$ is a vowel, and $\mathrm{N}$ is a sonorant consonant. The first step is to build a finite state representation of this dataset, which is called a prefix tree transducer (PTT). This PTT provides the correct output string for all and only the input strings in the dataset.

Presumably, acquiring the process the data represents means being able to provide an output string for any input (i.e, the domain of the target function is more than just the input strings that have been observed so far). Thus, in order to generalize beyond the dataset, the learner merges states in the PTT until it converges on the ISL FST in Figure 1.

The proof that it will converge relies on its state merging criterion: all and only states reached on the same sequence of $k-1$ input segments are merged. In this way the learner encodes an ISL bias that delimits the class of functions it can learn. The implication is that if the human phonological learner employs a similar bias, we have an explanation for why processes have the property of Input Strict Locality: only processes with this property are learnable. 
6. Discussion As mentioned above, the central finding of this paper that a large proportion of phonological processes have the computational property of Input Strict Locality has implications for both rule- and constraint-based theories of generative phonology, because the property is shown to hold of the input-output mapping regardless of the grammatical formalism used to describe it. The theoretical implication is that Input Strict Locality delimits the range of processes that the theory needs to describe, and, by extension, the range it should predict. This increased understanding of phonological possibility can inform our proposals for rules, constraints, and the ways they interact in the grammar.

7. Conclusions Modeling phonological processes with ISL functions demonstrates that Input Strict Locality is a defining property of the underlying input-output mapping. This property is useful in at least two ways. One, it delimits the range of processes we find and should expect to find in natural language phonology. Two, it serves as an inductive principle for a phonological learner generalizing the process from a finite number of positive examples.

\section{References}

Baković, Eric. 2013. Blocking and complementarity in phonological theory. Bristol, CT: Equinox. Blevins, Juliette. 2004. Evolutionary phonology. Cambridge: Cambridge University Press.

Chandlee, Jane. 2014. Strictly local phonological processes. Ph. D. thesis, University of Delaware.

Chandlee, Jane, Rémi Eyraud, and Jeffrey Heinz. 2014. Learning strictly local subsequential functions. Transactions of the Association for Computational Linguistics, to appear.

Chomsky, Noam and Morris Halle. 1968. The sound pattern of English. New York: Harper \& Row.

Hayes, Bruce, Robert Kirchner, and Donca Steriade. 2004. Phonetically based phonology. Cambridge: Cambridge University Press.

Heinz, Jeffrey. 2007. The inductive learning of phonotactic patterns. Ph. D. thesis, University of California, Los Angeles.

Heinz, Jeffrey. 2009. On the role of locality in learning stress patterns. Phonology 26. 303351.

Heinz, Jeffrey. 2010. Learning long-distance phonotactics. Linguistic Inquiry 41. 623661.

Hume, Elizabeth and Keith Johnson. 2001. The role of speech perception in phonology. San Diego: Academic Press.

Johnson, Mark. 1984. A discovery procedure for certain phonological rules. Proceedings of the 10th International Conference on Computational Linguistics. 344-347.

Kaplan, Ronald and Martin Kay. 1994. Regular models of phonological rule systems. Computational Linguistics 20. 371-387.

Koskenniemi, Kimmo. 1983. Two-level morphology: A general computational model for word-form recognition and production. University of Helsinki: Department of General Linguistics.

Legendre, Geraldine, Yoshiro Miyata, and Paul Smolensky. 1990. Harmonic grammar: A formal multi-level connectionist theory of linguistic well-formedness: Theoretical foundations. Cognitive Science Society. 388-395. Lawrence Erlbaum Associates.

McNaughton, Robert and Seymour Papert. 1971. Counter-free automata. Cambridge, MA: MIT Press.

Mielke, Jeff. 2008. The emergence of distinctive features. Oxford: Oxford University Press.

Prince, Alan and Paul Smolensky. 1993. Optimality theory: Constraint interaction in generative grammar. Technical Report RuCCS-TR-2, Rutgers University Center for Cognitive Science.

Rogers, James, Jeffrey Heinz, Margaret Fero, Jeremy Hurst, Dakotah Lambert, and Sean Wibel. 2013. Cognitive and sub-regular complexity. In Glyn Morrill and Mark-Jan Nederhof. (eds.), Formal grammar: lecture notes in computer science, vol. 8036, 90-108. Springer.

Rogers, James and Geoffrey Pullum. 2011. Aural pattern recognition experiments and the subregular hierarchy. Journal of Logic, Language and Information 20. 329-342. 